\title{
TRANSFORMACIÓN URBANA Y CAMBIO FUNCIONAL EN EL SUROESTE DE BURGOS: DE ESPACIO INDUSTRIAL A CAMPUS UNIVERSITARIO
}

Gonzalo ANDRÉS LÓPEZ

Departamento de Geografia.Universidad de Valladolid

Actualmente Burgos puede considerarse incluida en el nutrido grupo de ciudades españolas que forman el nivel intermedio del sistema urbano de nuestro país. Como núcleo de tamaño medio, ha estado afectada en los últimos años por una serie de problemas y oportunidades inherentes a la nueva manera de entender el desarrollo de la ciudad en la década de los noventa. Buena parte de los retos a los que se enfrenta han quedado definidos por la necesidad de renovar y valorizar espacios urbanos tradicionales que con el paso del tiempo han ido deteriorándose. La aparición de nuevas opciones en el desarrollo del núcleo y la existencia de numerosos problemas en su ordenación no han sido sino un claro ejemplo del carácter abierto de los procesos urbanos.

No debemos olvidar que las ciudades son los elementos del territorio en los que podemos distinguir un mayor dinamismo y una mayor capacidad de cambio, en función de la evolución permanente que ha llevado a considerarlas como verdaderos elementos "vivos" del espacio. Sin embargo, hasta mediados de siglo, este dinamismo no había formado parte del crecimiento en muchos de los núcleos que hoy entendemos como espacios urbanos consolidados. Buena parte de las capitales de provincia españolas coincidían entonces con pequeños núcleos urbanos desarrollados en función del aporte administrativo de la propia capitalidad provincial asignada en el siglo XIX, siendo realmente escasos los procesos en los que las ciudades de este tamaño demostraban un verdadero auge.

Burgos no ha sido una excepción y, en realidad, el crecimiento efectivo de la ciudad se producirá en los años 60 y 70 de nuestro siglo, momento en el que la recomposición del medio rural, y por consiguiente de sus estructuras sociales y económicas, y las posibilidades aportadas por el desarrollo industrial se configurarán como verdaderos factores de progresión urbana. Esta etapa desembocará en la conformación de una verdadera ciudad media en la que la estructura urbana ofrece un nivel de evolución suficiente como para poder analizar los fenómenos de cambio producidos en su interior.

Así ha ocurrido en la mayoría de las ciudades medias de nuestro país y, de manera específica, en las capitales de provincia de Castilla y León donde el estudio de los procesos de transformación urbana no ha podido acometerse hasta que el tamaño de los núcleos ha permitido su valoración sistemática. La norma ha sido considerar que, a la existencia de procesos de cambio urbano en un determinado

Polígonos, nº 9, 1999, pp. 9-29. 
espacio, le es inherente una serie de transformaciones inducidas en relación con el uso del suelo, la función en la ciudad, e incluso, su propia composición social (Álvarez Mora, 1978). En realidad, se trata de un proceso en el que la evolución urbana se traduce en el cambio de uso como verdadera manifestación del fenómeno, pudiendo producirse después el cambio de función y la reforma social de ese espacio. Esta evolución ha podido manifestarse en dos sentidos: por un lado, el de la renovación urbana y, por otro, el de la simple transformación, conceptos que aluden a procesos de cambio urbano pero entre los cuales cabe establecer alguna matización.

Por lo que respecta a la renovación urbana, el proceso de cambio supone la aparición de un nuevo uso urbano sobre una función deteriorada o en proceso de recomposición, mientras que la transformación alude a aquellos casos en los que la sustitución de usos se realiza sobre una actividad plenamente activa o un espacio sin función aparente al que se dota de sentido urbano.

Ahora bien, tanto los procesos de transformación urbana como los casos en los que se puede hablar de renovación han de entenderse sobre la base de un sector de la ciudad preexistente, consolidado, lo cual no significa la total continuidad en la ocupación física del espacio. Precisamente, algunos de los procesos de transformación y/o renovación urbana existentes en ciudades medias se han producido en los espacios suburbanos en los que coinciden de manera peculiar barrios tradicionales, asentamientos industriales, fincas de cultivo, equipamientos urbanos... y, en definitiva, toda una serie de actividades específicas.

Esta singular composición del espacio y la existencia de un proceso de renovación y transformación han coincidido en el desarrollo urbano de Burgos en los últimos quince años. Concretamente, el Suroeste de la ciudad, entendiendo por tal espacio aquel situado al Norte del ferrocarril, al Sur del río Arlanzón, al Oeste de la Plaza de Castilla y al Este del barrio de La Sesa como límite occidental incluido en este conjunto, se ha visto afectado por un proceso de cambio urbano en el que se han manifestado actuaciones de renovación y de transformación sobre la base de una orientación funcional muy definida. El objetivo de este trabajo es, por tanto, acometer una pequeña valoración acerca de los fundamentos de este proceso de cambio en el que el suroeste de Burgos se encuentra plenamente inmerso.

Para ello debemos tratar, en primer lugar, los factores fundamentales que explican la estructura urbana de Burgos y el papel singular del Suroeste en el conjunto de la ciudad. Y es que la tradicional vinculación del núcleo con las actividades militares, de transporte y comunicaciones, a la par que su propia localización geográfica en una encrucijada de caminos vinculada a la Ruta Jacobea, han supuesto la formación de una particular estructura urbana definida por los propios elementos del medio físico.

El asentamiento inicial del núcleo en la falda del Cerro de San Miguel y la situación en el valle del río Arlanzón son una base sobre la que Burgos se ha ido desarrollando a lo largo de su formación como ciudad. A ésta se le añade, de ma- 
Plano 1. El suroeste de la ciudad de Burgos.

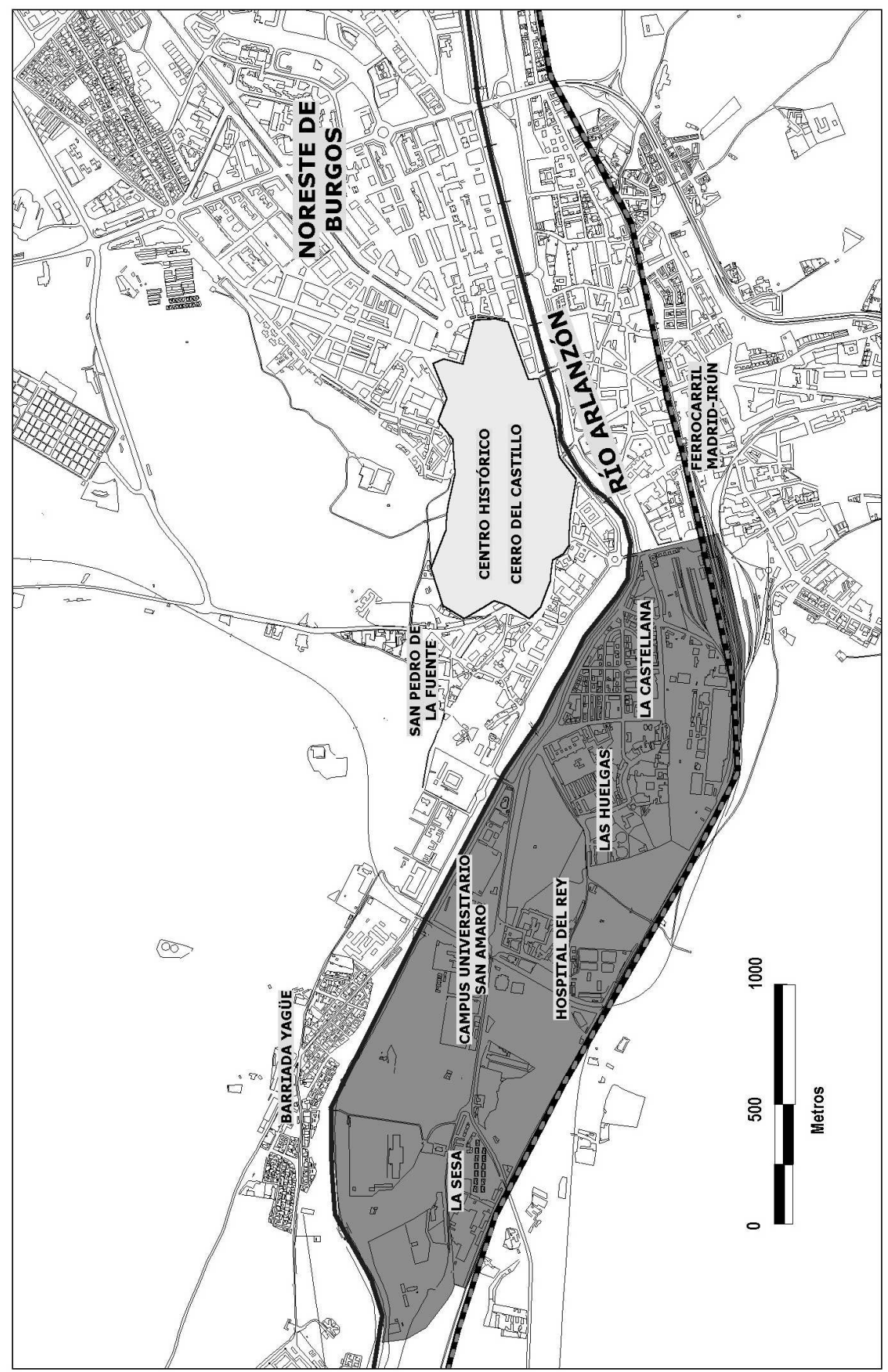


nera temprana, la instalación de las vías del ferrocarril coincidiendo, por tanto, tres elementos directores del crecimiento urbano en torno al centro del Burgos tradicional. Cuando desde finales de los años 50 comience el verdadero desarrollo y la necesidad de vivienda donde alojar a la población se manifieste de manera apremiante, el significado de estos condicionantes será patente en la configuración del núcleo. La coincidencia del centro histórico con el río Arlanzón, el Cerro de San Miguel y las vías del ferrocarril en un espacio lineal de apenas un kilómetro de distancia definirá una barrera urbana difícil de superar para la ciudad. Ante la existencia de este límite, Burgos crecerá en el extenso espacio que, al Este del centro histórico y al Norte del río, le proporcionaba la vega natural del propio Arlanzón.

La ciudad ha tenido, por tanto, dos límites muy definidos aportados por los caracteres físicos del espacio ya que el río se ha considerado como el límite NorteSur del núcleo, mientras que el conformado por el cerro y el propio río, junto con las vías férreas, ha explicado las diferencias Oeste-Este. En el marco de esta configuración, el Suroeste ha sido un espacio urbano en espera a lo largo de muchos años. Dada su ubicación con respecto al centro histórico y en función de una topografía favorable a la construcción parecía lógica su ocupación en los años 60 y 70 cuando Burgos crece de manera importante tras ser declarada Polo de Promoción Industrial en el año 1964. Sin embargo, la conjunción de una serie de factores en relación con el uso del suelo, la ocupación tradicional, el planeamiento urbano y los intereses de la propiedad en otros sectores de la ciudad harán que la colonización definitiva de este conjunto no llegue hasta los años 90 .

En realidad, podemos decir que el Suroeste de la ciudad se ha caracterizado, hasta este momento, por ser un espacio ocupado por usos marginales, que tienen una función específica en el núcleo, pero manifiestan unas necesidades de terreno y ubicación muy particulares. La organización del espacio urbano hay que buscarla en el significado de los barrios de Huelgas y Hospital del Rey como elementos vertebradores del conjunto desde la época medieval. Sin embargo, la orientación funcional vendrá definida por la actividad industrial y el asentamiento de usos del suelo vinculados al equipamiento de la ciudad.

\section{LA INDUSTRIA Y LOS EQUIPAMIENTOS COLECTIVOS COMO LOS DOS USOS TRADICIONALES DE UN CONJUNTO SINGULAR DE BURGOS.}

Por lo que respecta a la actividad industrial, dos hechos pueden considerarse: por un lado, la instalación de las primeras plantas fabriles de la ciudad aguas abajo del Hospital del Rey desde el año 1841, momento en el que inicia su actividad la Fábrica de Papel Continuo y, por otro, la llegada a Burgos del ferrocarril en el año 1860. Ambas efemérides supondrán los pilares de la caracterización del Suroeste como verdadero espacio industrial. La vocación fabril definida por la fábrica de papel se continuará con la instalación de la Sociedad Española de Seda Artificial (SESA) en los años 30 creando un barrio obrero que será otra de las piezas singulares de este conjunto ya que la factoría llegará a contar con más de 800 empleados en plantilla. 
En los años 40, el Plan de Ensanche de Paz Maroto regularizará la orientación fabril clasificando como suelo industrial prácticamente todo aquel que en este espacio no había sido ocupado, ya que la ciudad se había forjado en un marco en el que no existían aún normas detalladas de planificación urbana ${ }^{1}$. La instalación de la Cellophane Española en el año 1949 y su declaración como Industria de Interés Local marcarán el camino definitivo que seguirán otras plantas industriales como las de Bakimet o Productos Químicos SA, que no harán sino terminar de dibujar la configuración de este sector de la ciudad como la verdadera célula industrial del núcleo ${ }^{2}$.

El espacio no ocupado por la industria será destinado a la instalación de diversas actividades vinculadas al servicio colectivo de Burgos. Siguiendo la pauta marcada por la construcción del Hospital Militar de la ciudad en el año 1887, el propio Plan de Ensanche clasificará suelo destinado a recoger actividades singulares. En este marco se explica la construcción del Mercado de Ganados de San Amaro y la Unidad Técnica de Veterinaria Militar que no harán sino aportar una mayor especialización del espacio en los usos marginales respecto al dominio meridiano ejercido por la residencia en el resto del núcleo urbano ${ }^{3}$.

La conjunción de este tipo de actividades específicas y de la industria, en el conjunto del Suroeste, con los barrios rurales de Huelgas y Hospital del Rey y con el suburbio jardín de La Castellana generarán un espacio caracterizado por el uso marginal respecto a la ciudad (ANDRÉS LÓPEZ, 2000: 49). Nazario González lo valoraba de manera muy acertada al explicar cómo arrancando del “...diseminado agrícola de las Huelgas y el Hospital del Rey..." se había conformado un conjunto diverso y difícil de organizar ya que "...casas de labradores, chalets familiares con su jardín, naves de escasa altura..." únicamente contribuían a generar una baja densidad de población (GONZÁLEZ, 1958: 271).

Como consecuencia de todo ello, cuando en el año 1970 se apruebe el primer Plan General de Ordenación Urbana de Burgos, documento conocido como Plan García Lanza, el suroeste de la ciudad estará aglutinado por una función específica dentro del núcleo pero se definirá por ser un conjunto ocupado por actividades que necesitan grandes paquetes de suelo y en el que la desarticulación interna es la norma. Este documento de planeamiento no solucionará, sin embargo, la falta de coherencia interna del espacio pues entenderá que la actividad industrial y los equipamientos colectivos pueden seguir siendo los elementos vertebradores del te-

\footnotetext{
${ }^{1}$ Hasta que en el año 1944 se aprueba el Plan de Ensanche y Reforma Interior de la ciudad, en Burgos no habían existido otras normas de planificación urbana que las obsoletas Ordenanzas Municipales de 1747, 1849 y 1885, apoyadas en algún caso por Bandos de Policía Urbana y Rural hasta el año 1929. De ahí que la regularización de buena parte de las orientaciones en el uso del suelo de los sectores de Burgos llegase por parte del Plan de Ensanche de Paz Maroto. Archivo Municipal de Burgos, Administración, 4.031/1, 4.032/1 y 4.033/1.

${ }^{2}$ En el año 1949 se declara a la Cellophane Española Industria de Interés Local (Archivo Municipal de Burgos, Fomento, 1.108) dada la importante aportación realizada respecto a la vida económica y social del municipio. La planta supondrá una inversión de más de 60 millones de pesetas dando empleo a 400 trabajadores (Archivo Municipal de Burgos, Obras Particulares, 12.449).

${ }^{3}$ El 3 de Abril de 1946 en sesión extraordinaria del Ayuntamiento Pleno se aprueba el traslado del Mercado de Ganados de su antigua ubicación en San Lucas a los terrenos de San Amaro. Archivo Municipal de Burgos. Obras Públicas, 3.597.
} 
rritorio en un marco de crecimiento industrial que justificaba tal decisión (MANERO Miguel, 1982).

La planificación de polígonos industriales de nueva factura (Gamonal y Villalonquéjar) y sus ventajas de accesibilidad y facilidad de instalación para las empresas harán que la industria en el Suroeste pierda su entidad dada la crisis de la mayor parte de las fábricas que en este espacio se habían instalado y la inexistencia de nuevas implantaciones. Como consecuencia de ello, en los años 70, este sector de Burgos se caracterizará por la conjunción de una serie de factores en relación con el abandono y la degradación del espacio urbano. Esta situación, a la que hay que añadir la recomposición de la actividad rural que ocupaba buena parte del conjunto, conllevó la necesidad de una serie de actuaciones de reforma que se pueden entender como el inicio de la renovación y transformación urbana que vamos a estudiar en los años 90 .

De hecho, la instalación por parte del propio Ayuntamiento de Burgos de algunos equipamientos deportivos y la cesión de los terrenos por la corporación municipal para instalar el Colegio Universitario Adscrito de Burgos (CUA), como consecuencia de una remodelación efectuada sobre los usos tradicionales del espacio, van a definir el inicio de la transformación urbana del Suroeste y van a ser el elemento clave para que se desvincule a éste de su tradicional vocación industrial. La construcción del Colegio Universitario como culminación de un largo proceso iniciado en 1971 y la remodelación efectuada sobre la parcela del Mercado de Ganados supondrán, efectivamente, la primera actuación de renovación urbana. En el año 1978 se demolió el Mercado de Ganados para su traslado al cercano paraje de la Milanera y un año después se derribaron también los edificios de Veterinaria Militar con el objeto de la construcción del complejo deportivo municipal de San Amaro, que se inició en el año 1979 con la dotación de la actual pista de atletismo.

No obstante, la base de estas transformaciones se normalizará con la entrada en vigor del Plan General Delta Sur en el año 1985. Será con este documento con el que el Suroeste perderá su tradicional ligazón a la actividad fabril al entender la ciudad como un conjunto de actividades integradas en el que la segregación funcional no tiene cabida. En relación con esta concepción, se introducirá la previsión del crecimiento urbano mediante la construcción de viviendas y la alternancia de usos, en un contexto en el que el deterioro del espacio había llegado a unos niveles en los que se exigía la intervención urbana (ANDRÉS LÓPEZ, 1999: 446).

La degradación de las actividades rurales en los barrios de Huelgas y Hospital del Rey había conllevado el abandono de varias de las viviendas iniciándose así un proceso de deterioro del inmovilizado que en algunos casos ha llegado hasta la actualidad (Plaza del Sobrado). La necesidad de actuar sobre el conjunto del Suroeste se ponía de manifiesto en la existencia de diversas propuestas para la recuperación de estos barrios tradicionales que se iniciaron con la solicitud de puesta en marcha de un Plan Especial de Remodelación del Hospital del Rey por parte del Consejo de Administración del Servicio Municipalizado de Desarrollo Urbano y Fomento de la Construcción del Ayuntamiento de Burgos, la cual quedó sin 
Figura 1. Plan Parcial "Parral" y Plan Parcial "Sedera" en el suroeste de Burgos.
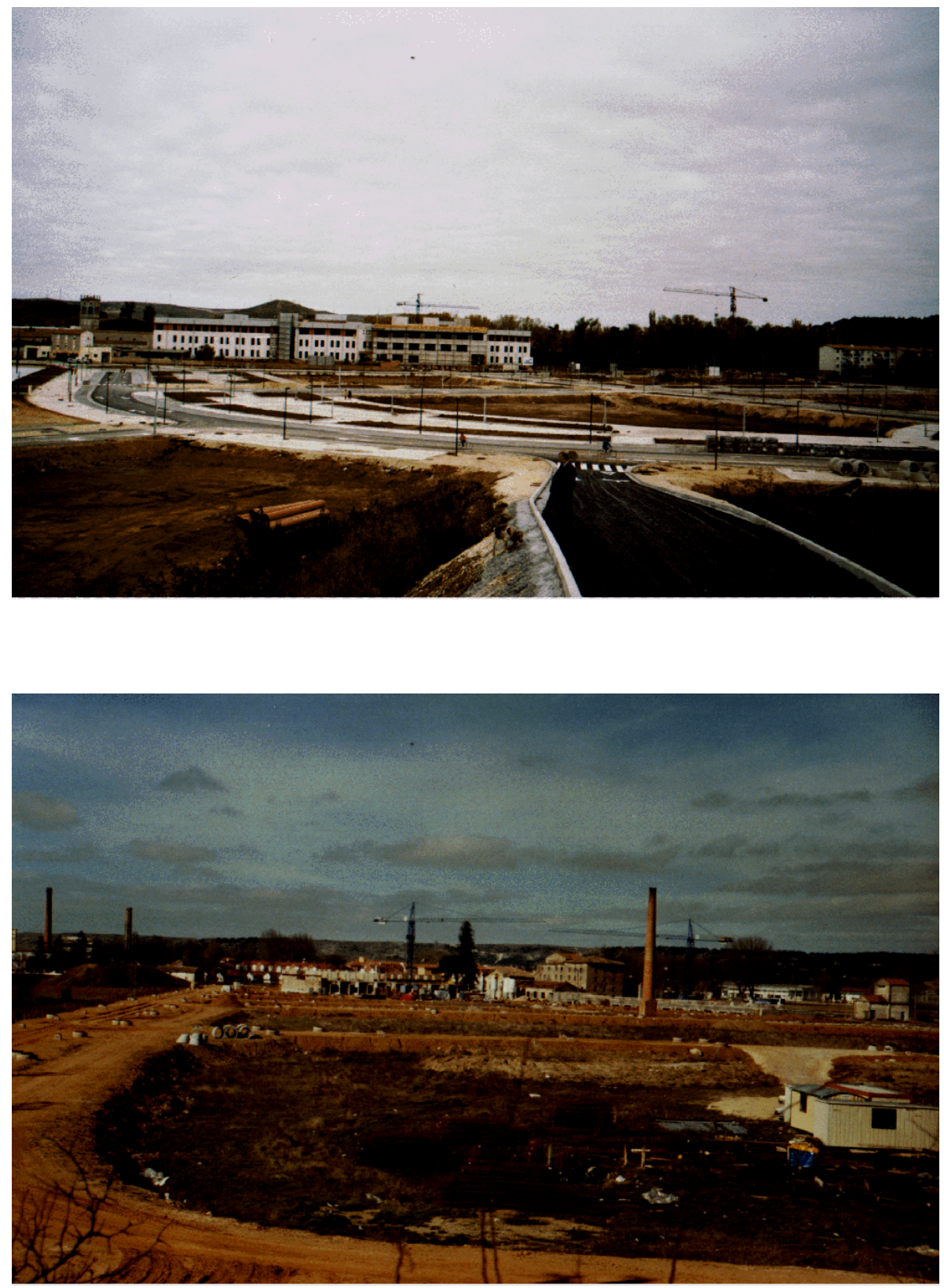
desarrollar en virtud de la inclusión de este barrio y del de Las Huelgas en el primer borrador del Plan Especial del Centro Histórico de Burgos ${ }^{4}$. Con todo, las intervenciones a realizar sobre un espacio en proceso de deterioro tanto desde el punto de vista meramente físico como desde la perspectiva social y funcional habían de producirse. Por un lado, se planificarán sendos Planes Parciales para la construcción de viviendas con el fin de incorporar a la ciudad aquellos espacios del Suroeste aún sin ocupar (Plan Parcial Parral y Plan Parcial Sedera) 5 . Por otro, se producirá el fracaso definitivo de la previsión urbana con respecto a la actividad industrial puesto que nunca se llevará a efecto el denominado Plan Parcial Industrial - Comercial en el que, como resumen de la idea de integración que impregnaba todo el PGOU, se hacían compatibles industria y comercio como complemento de la actividad residencial.

Finalmente se optaría por una especialización funcional del espacio concreta al hacer efectiva la idea de dotar a Burgos de un conjunto plenamente universitario. En consonancia con esta idea se procedió a la firma de un convenio entre el Ayuntamiento de Burgos, la Universidad de Valladolid, la Junta de Castilla y León, la Diputación Provincial de Burgos y el Ministerio de Educación y Ciencia, mediante el cual se llevaba a cabo la recuperación del Hospital del Rey con el fin de destinar el edificio a Facultad de Derecho ${ }^{6}$.

La conversión del antiguo hospital en facultad universitaria será, en realidad, el factor definitivo sobre el que se pueda entender el proceso de transformación y renovación urbana en los años 90. La suspensión del Plan General Delta Sur y el inicio de su revisión con el fin de elaborar el actual PGOU no serán sino un elemento más que apoyará el abandono de la tradicional visión industrial de este espacio que desde comienzos de esta década ha estado definitivamente afectado por la dinámica de cambio urbano ${ }^{7}$.

\section{DE ESPACIO INDUSTRIAL A CAMPUS UNIVERSITARIO: LA TRANSFORMACIÓN URBANA DEL SUROESTE DE LA CIUDAD.}

Son dos los factores sobre los que puede interpretarse este proceso de cambio que, actualmente, se encuentra todavía abierto. Por un lado, el papel de la función

\footnotetext{
${ }_{5}^{4}$ Archivo Municipal de Burgos, AD-5.096/10.

${ }^{5}$ Plan General de Ordenación Urbana Delta Sur. Servicio de Urbanismo. Ayuntamiento de Burgos. 1985 y 1992.

${ }^{6}$ La recuperación efectiva del Hospital, en una de las actuaciones de renovación urbana más importantes llevadas a cabo en Burgos, se llevó a efecto mediante la firma del convenio el 26 de octubre de 1987, añadiéndose una addenda el 21 de marzo de 1989. Archivo Municipal de Burgos. $\mathrm{AD}-2.952 / 14$.

${ }^{7}$ La sentencia del Tribunal Supremo de 31 de julio de 1991 supuso la suspensión efectiva del Plan General Delta Sur cerrando así un proceso abierto desde su aprobación en 1985. Desde el 15 de abril de 1983, fecha en la que se aprueba inicialmente el Plan, hasta 1985 se realiza su primera exposición pública. Sin embargo, entre esta exposición y su aprobación definitiva, se producen modificaciones importantes que hacen que el Plan sea definitivamente suspendido dada la inexistencia de la preceptiva segunda exposición pública. El documento será revisado y aprobado de nuevo el 23 de julio de 1992 para ser poco después iniciada su revisión que desembocará en el Plan General actual. Servicio de Urbanismo del Ayuntamiento de Burgos.
} 
universitaria por cuanto que ésta tiene una serie de necesidades espaciales y de infraestructura que se han comenzado a llevar a cabo desde la creación de la Universidad de Burgos en el año 1994. Por otro, la materialización efectiva del desarrollo mediante la urbanización del espacio y la consiguiente construcción de viviendas inherente a la ejecución definitiva de los Planes Parciales Parral y Sedera.

Sin embargo, hay que tener en cuenta previamente, que la sustituciónrenovación de usos o la incorporación de nuevas actividades a la ciudad, en el marco de un espacio con piezas consolidadas, ha de ser respetuosa con lo preexistente en aras de conjugar la tradición con las nuevas funciones urbanas. En realidad, un proceso de cambio ha de entenderse como una de las manifestaciones del dinamismo de la ciudad, pero no por ello ha de implicar la sustitución total de usos o funciones ya que la destrucción de los espacios urbanos con raíz histórica no lleva sino a favorecer el incremento de la memoria de la ciudad.

En el caso que nos ocupa, la aparición de nuevas viviendas se ha realizado sobre fincas rurales no incorporadas hasta entonces a la urbanización. Por el contrario, la implantación sistemática del uso universitario ha manifestado una mayor relación con los espacios urbanos del Suroeste ya consolidados. La imbricación entre la Universidad, como factor de cambio urbano, y los barrios del Suroeste, como elementos preexistentes, ha quedado teóricamente regulada con la incorporación de algunos de éstos barrios al Plan Especial del Centro Histórico de Burgos.

El PECH de Burgos comenzó a redactarse en el marco de la elaboración del Plan General de 1985 por parte de la empresa Delta Sur SA llegándose a aprobar inicialmente un primer texto el 22 de Enero de 1990. Transcurrido un lapso de tiempo desde esta aprobación, el Ayuntamiento de la ciudad encomendará de nuevo la redacción del documento, esta vez a la empresa PROINTEC SA, encargada de la elaboración del nuevo Plan General. Este segundo documento se aprobará definitivamente el 28 de Abril de 1995 recogiendo buena parte de la estructura del primer borrador del PECH pero adaptando su visión orgánica a la del nuevo Plan General $^{8}$.

En su delimitación definitiva, el PECH de Burgos no se ceñirá exclusivamente al centro histórico, entendido en su visión más tradicional, sino que otorgará una atención especial a dos barrios históricos de Burgos como son Las Huelgas y el Hospital del Rey. De manera general se pretende la puesta en valor del tejido histórico de ambas unidades mediante la conservación y la revitalización. Como objetivos específicos en relación con esta premisa, podemos señalar la necesidad de fijar a la población en su hábitat tradicional, la conservación del patrimonio arquitectónico, monumental e histórico, el mantenimiento de la estructura urbanística existente, la peatonalización y la ordenación de espacios carentes de planificación.

El desarrollo de estos objetivos ha quedado definido en la existencia de una serie de propuestas concretas que, a su vez, se han centrado sobre las premisas establecidas por el Plan en cuanto a la ordenación y a la protección del espacio urba-

\footnotetext{
${ }^{8}$ Oficina del Centro Histórico. Ayuntamiento de Burgos. Plan Especial del Centro Histórico de Burgos. 1995. Memoria.
} 
no. Por lo que respecta a esta última, el PECH ha delimitado tres niveles de protección para el patrimonio edificado (Integral, Estructural y Ambiental), desde el mero mantenimiento de las fachadas y elementos originarios a la conservación integral de los edificios según el valor otorgado a cada uno de ellos?

Figura 2. Edificio catalogado por el PECH de Burgos con el grado de protección ambiental.

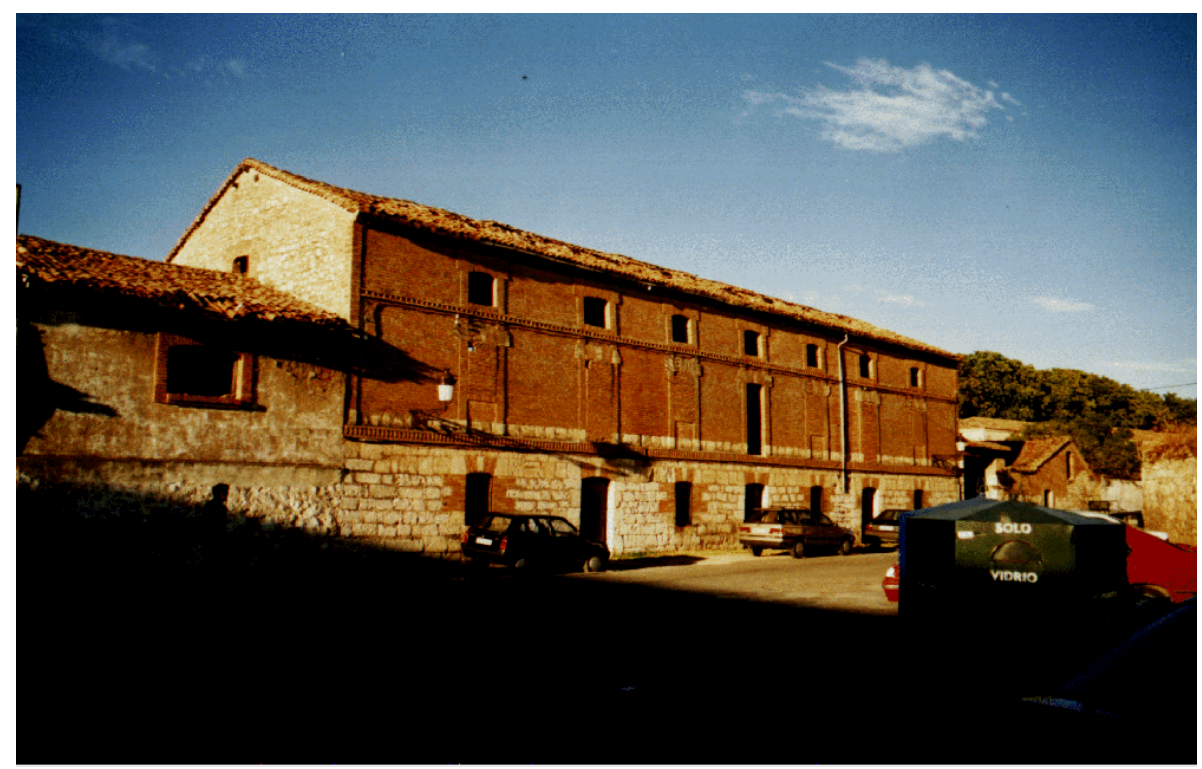

La Protección Integral permite únicamente las actuaciones de conservación dado el interés en mantener el valor histórico-artístico de los edificios y la obligación de no modificar las condiciones referentes a fachadas, estructuras, organización interna, composiciones de color, ornamentos o cubiertas. Bajo esta norma de protección se ha englobado a edificios singulares en el suroeste de la ciudad como la Ermita de San Amaro, el Arco de la Villa, el Arco de la Plaza del Compás, la Iglesia de San Antonio en el barrio de Huelgas, la puerta Este del Parque del Parral y, obviamente, el propio Monasterio de las Huelgas y el edificio del Hospital del Rey.

La Protección Estructural autoriza obras de conservación y restauración en función del interés por mantener los elementos compositivos y estructurales primigenios. La norma permite, por tanto, modificar la estructura interna de los edificios siempre y cuando estos mantengan las fachadas y la estructura exterior además del resto de elementos fundamentales del inmueble (cubierta, ornamen-

\footnotetext{
${ }^{9}$ Oficina del Centro Histórico. Ayuntamiento de Burgos. Plan Especial del Centro Histórico de Burgos. 1995. Catálogo de Elementos, Espacios y Edificios Protegidos.
} 
tos...). Bajo este nivel de protección se ha incluido la mayoría de los edificios de la Plaza del Compás y el Hospital Militar de Burgos.

Finalmente, la Protección Ambiental ha tenido como objetivo la conservación de fachadas y elementos originarios autorizándose las obras de reforma y rehabilitación, lo cual se ha materializado en que se permita la modificación de la estructura y organización interna de los inmuebles a costa de la conservación de los elementos exteriores. A este nivel de protección se corresponden la mayor parte de las viviendas de la Calle Alfonso VIII y algunos de los edificios del Hospital del Rey.

Teniendo en cuenta los tres niveles, el PECH de Burgos ha establecido algún tipo de protección en 50 edificios del suroeste de la ciudad lo que, de partida, supone un marco de referencia para cualquier tipo de actuación urbana sobre este espacio. No obstante, el papel del Plan como marco regulador de la transformación no se ha limitado al establecimiento de unos niveles de protección sobre los edificios existentes sino que ha especificado una serie de actuaciones a desarrollar con el fin de cumplir los objetivos de conservación y revitalización.

Estas propuestas de ordenación se han estructurado en tres niveles de intervención: por un lado las denominadas Unidades de Ejecución (UE), con las que se pretende la ordenación conjunta de espacios concretos; por otro las llamadas Áreas de Intervención (AI) con las que se persigue fomentar la posibilidad de que la iniciativa pública desarrolle intervenciones destinadas a mejorar las condiciones medioambientales y estructurales; finalmente las Intervenciones Puntuales (IP) con el sentido de poder llevar a cabo pequeñas actuaciones encaminadas a objetivos concretos ${ }^{10}$. Por lo que respecta a los barrios de Huelgas y Hospital del Rey, el Plan no ha previsto ninguna IP pero sí ha planificado diversas Unidades de Ejecución y Áreas de Intervención que ponen de manifiesto la dinámica de cambio urbano que afecta a este espacio de Burgos.

Las siete Unidades de Ejecución previstas en el Suroeste se han dirigido en dos sentidos: por un lado, se han encaminado a reorganizar el tejido urbano preexistente con actuaciones para generar nuevos viales o nuevas construcciones en espacios sin ocupar o con una ordenación deficiente y, por otro, se han centrado en actuaciones de renovación urbana con las que se pretende la sustitución de un uso en declive. Mientras la primera tipología se centra en cuatro UE de escasas dimensiones, la renovación urbana se manifiesta en las tres restantes, de mayor entidad. En estas tres actuaciones se ha previsto la reconversión de la Plaza del Sobrado, la sustitución del Acuartelamiento Juan Yagüe por bloques de vivienda y la reorganización del Parque de Maquinaria propiedad de la Diputación Provincial en el Hospital del Rey ${ }^{11}$.

\footnotetext{
${ }^{10}$ Oficina del Centro Histórico. Ayuntamiento de Burgos. Plan Especial del Centro Histórico de Burgos. 1995. Memoria. p 7.

${ }^{11}$ La reconversión y recuperación de la Plaza del Sobrado se inició el 27 de Enero de 1999 con la firma de un Convenio de Colaboración entre el Instituto Universitario de Restauración (IUR) de la Universidad de Burgos y el Ayuntamiento de Burgos para la realización de un estudio de propuestas sobre reforma, rehabilitación y revitalización de este espacio. La sustitución del Acuartelamiento Juan Yagüe comenzó con la elaboración del Proyecto de Reparcelación Voluntaria por parte del Ministerio de Defensa y la venta de la parcela por un valor de casi 1.000 millones de pe-
} 
Por lo que respecta a las cinco Áreas de Intervención de iniciativa pública, se ha planificado la creación de nuevos viales (traseras calle Juan de Austria) y la urbanización de algunos espacios (Paseo de la Universidad), prestando especial atención a la necesidad de integrar la Universidad en el entorno de la Plaza del Sobrado. A tal efecto se introdujo en el Plan un Área de Intervención específica (5.AI.2) que permitiese la construcción de dos nuevos edificios previstos en el desarrollo de la Universidad de Burgos, para lo cual fue necesario la puesta en marcha del Plan Especial de la Universidad ${ }^{12}$.

Este organismo público ha sido, de hecho, uno de los agentes con mayor peso en el proceso de transformación y renovación urbana del Suroeste del núcleo ya que, sobre la base del actual PGOU de Burgos y con el marco regulador establecido por el PECH, ha iniciado una serie de actuaciones encaminadas a conseguir la construcción de un verdadero campus universitario. La idea de concentrar todos los centros universitarios en un único espacio de la ciudad parte de la creación de la propia Universidad en el año 1994 y, en realidad, se ha constituido como su pilar ideológico respecto al tratamiento del espacio urbano y en relación a su integración dentro de Burgos.

La puesta en marcha de este modelo parte de la ya comentada recuperación del Hospital del Rey y la construcción de la nueva Facultad de Humanidades en el año 1990, pero su concreción no ha tenido efecto hasta la revisión definitiva del Plan General de la ciudad ${ }^{13}$. El propio Vicerrectorado de la Universidad era consciente de que el Plan de 1985 no estaba diseñado para asumir la función universitaria en un planteamiento similar a éste, ya que se consideraba que aquel documento "consolida las dotaciones universitarias existentes, pero no prevé nuevas dotaciones de suelo"14.

Con el objetivo de desarrollar la creación de un auténtico espacio universitario, la UBU presentó en septiembre de 1997 una serie de alegaciones complementarias a la revisión del Plan General de Ordenación Urbana, las cuales se acompañaban de un completo documento en el que se recogían las aspiraciones del modelo universitario. Bajo un singular soporte ideológico resumido en la máxima de "un Burgos estable, en una España estable, en una Europa estable, en una economía globalizada", se requería al planeamiento la reserva de una importante cantidad de suelo para desarrollar las necesidades de la Universidad como agente dinamizador de la vida cultural de Burgos ${ }^{15}$. El significado de este estudio es el de dotar a la UBU de una justificación pormenorizada de los beneficios del modelo de

setas. La última de las Unidades de Ejecución aún no ha comenzado a tramitarse. Ayuntamiento de Burgos. Oficina del Centro Histórico.

${ }^{12}$ El Plan Especial de la Universidad se aprobó definitivamente el 18 de junio de 1998 en ejecución del Área de Intervención 5.A.I.2 del PECH de Burgos con el fin de desarrollar las necesidades de equipamiento docente e investigador de la UBU. Ayuntamiento de Burgos. Servicio de Urbanismo. Exp. 2609/95.

${ }_{13}$ Archivo Municipal de Burgos, AD-4.736/2.

${ }^{14}$ Informe de Gestión 1994 - 1997. Vicerrectorado de Infraestructuras e Instalaciones. Universidad de Burgos. 1997. p 12.

${ }^{15}$ Documentación complementaria a la Alegación formulada por la Universidad de Burgos en el trámite de Información pública del Documento de Revisión del Plan General de Ordenación Urbana de Burgos. Septiembre de 1997. Unidad Técnica de la Universidad de Burgos. p 14. 
campus único y su desarrollo en lo que se denominará "Campus de El Parral San Amaro - La Milanera", ya que se pretende ocupar una importante cantidad de suelo para uso universitario.

Sobre la base de un espacio inicial de unas 40 hectáreas, este documento preveía la necesidad final de unos $800.000 \mathrm{~m}^{2}$ en un marco de inserción del campus de unas 400 ha. Con una reserva final de poco más de 30 hectáreas, la Universidad ha dejado ya patente su incidencia en la configuración del espacio urbano del Suroeste de la ciudad. Mediante el desarrollo del citado Plan Especial se han construido el edificio destinado a Biblioteca Central y la Facultad de Ciencias de la Empresa como actuaciones más importantes. Como operaciones secundarias, se han llevado a cabo la construcción del Polideportivo Universitario, junto a la Facultad de Humanidades, y la ampliación de la Facultad de Ciencias.

Pese a que las actuaciones llevadas a cabo por la Universidad aún son escasas lo cierto es que los proyectos en ejecución o en fase de preparación supondrán la ocupación de buena parte del espacio de este conjunto. La Residencia Camino de Santiago, reutilizando el antiguo Hostal de San Amaro, el nuevo Centro de Tecnologías de la Construcción, el edificio I+D y el Edificio de Servicios Centrales de la Universidad en la manzana de la Ermita de San Amaro son un claro síntoma de que el suroeste de la ciudad se encuentra en un fuerte proceso de cambio a todos los niveles.

Así lo demuestran las previsiones de urbanización y construcción del nuevo Plan General de Burgos ya que asignan a este conjunto un numeroso grupo de Planes Parciales a desarrollar con el fin de incorporar al mismo un número considerable de viviendas. Actualmente, se encuentran en ejecución el Plan Parcial Parral, que supone la incorporación de 728 nuevas viviendas, y el Plan Parcial Sedera que creará otras $565^{16}$. A ello hay que unirle las 624 viviendas de la Unidad de Ejecución Bakimet que tras años de gestiones parece estar definitivamente en marcha. Estas tres actuaciones suponen la creación de casi 2.000 viviendas a las que se unirán en un futuro otras 170 previstas en el Sector de Suelo Urbanizable S-25. Si tenemos en cuenta que en el Distrito Censal Número 8 de la ciudad, en el que se integra todo el Suroeste llegando hasta los límites del municipio, viven actualmente poco más de 2.000 personas podemos entender el significado que llegarán a tener los nuevos edificios pues supondrán, cuando menos, duplicar la población actual ${ }^{17}$.

Por otra parte, la transformación asociada a este conjunto no se limita a la mera recomposición en función de las necesidades universitarias y las actuaciones privadas en la construcción de viviendas sino que desde el propio planeamiento se recoge la idea de introducir modificaciones en el uso del suelo. Con tal sentido se

\footnotetext{
${ }^{16}$ Ambos planes son un legado del PGou Delta Sur y se han incluido en el Plan Actual como Áreas de Planeamiento Incorporado. Servicio de Urbanismo del Ayuntamiento de Burgos. Exp. $2.210 / 91$ y $2.791 / 91$.

${ }^{17}$ Padrón Municipal de Habitantes por Distritos y Secciones Censales de la Ciudad de Burgos. 1998. Sección de Estadística. Ayuntamiento de Burgos.
} 
Plano 2. Usos universitarios y viviendas. La nueva orientación funcional del suroeste de Burgos.

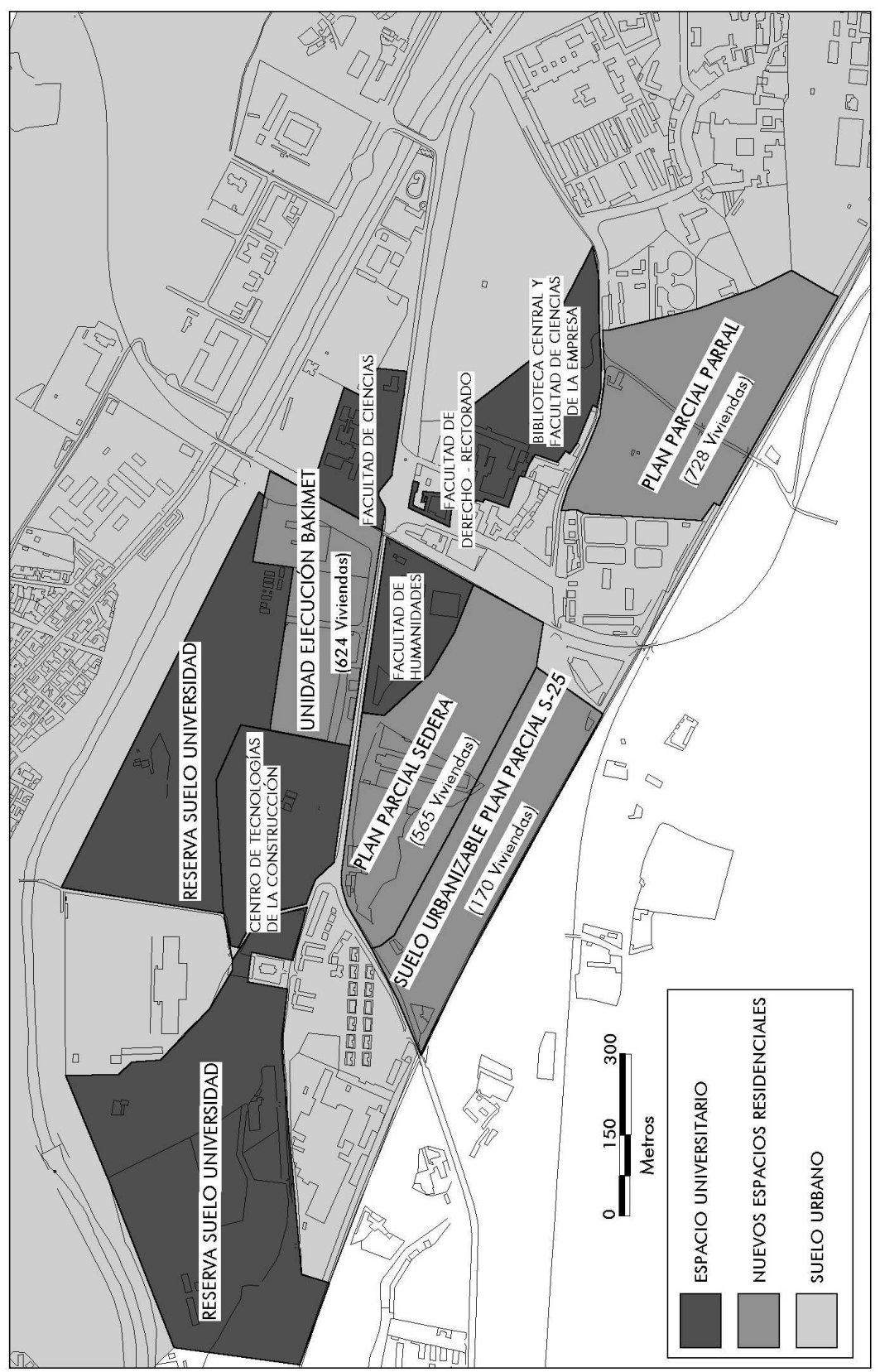


habilita en el Plan General la figura de las Áreas de Transforma ción que pretende desarrollar un fundamento de la planificación tradicional entendiendo que las actividades industriales han de relocalizarse progresivamente en los polígonos de nuestra ciudad. Bajo esta idea se pretende trasladar, en un plazo medio de tiempo, la fábrica de Cellophane Española, los depósitos de CLH, o los Almacenes de Continental Auto al polígono industrial de Villalonquéjar. Con ello, el Suroeste de Burgos quedará privado de los últimos vestigios industriales que aún continúan activos y las fábricas pasarán a ser una parte más de la historia de este espacio.

El conjunto de actuaciones hasta aquí comentadas está teniendo una influencia considerable, ya no sólo sobre lo que es el Suroeste en sí, sino en relación a la propia configuración de la ciudad. La renovación urbana que suponen algunas de ellas, caso del nuevo inmueble de Servicios Centrales de la UBU o la construcción de viviendas en el actual emplazamiento de Bakimet, se complementa con un profundo proceso de transformación en la mayoría del conjunto. El cambio de usos propiciado por la ejecución de los Planes Parciales o la construcción de edificios docentes indica una variación en la estructura urbana mucho mayor de lo que, a priori, puede parecer.

\section{EL SIGNIFICADO DE UN NUEVO ESPACIO EN LA ESTRUC- TURA GENERAL DE UN NÚCLEO URBANO DE TAMAÑO ME- DIO.}

El suroeste de la ciudad ha sido siempre un espacio en el que los usos peculiares con una personalidad específica han sido el rasgo distintivo. En los años 90, el nuevo desarrollo de este espacio y las necesidades de construcción de la Universidad han explicado una nueva estrategia de actuación de los agentes urbanos. Atrás queda la industria como elemento definitorio del conjunto, pero atrás queda también la posibilidad de que esta actividad conviva con los nuevos usos directores de la transformación. Si la marginalidad ocasionada por las fábricas fue un hecho manifiesto hasta los años 70, en la actualidad, se ha optado por una estrategia similar aislando los usos productivos en un claro ejemplo de segregación funcional heredada de la planificación rígida del espacio. Desde luego, la opción planteada por el Plan de 1985 en la que convivían la industria, el comercio y la residencia ha quedado definitivamente desechada.

El nuevo planeamiento de Burgos ha permitido la construcción de viviendas en el Suroeste pero lo cierto es que este hecho sólo ha llegado cuando la Universidad de la ciudad ha definido su estrategia espacial y los intereses de la promoción inmobiliaria han entendido a este agente público como un elemento de nueva centralidad urbana. La Universidad está siendo capaz de generar algunos efectos multiplicadores y ha centrado la atención de una parte importante de la promoción inmobiliaria en estos últimos años. La profusión de iniciativas para la construcción de edificios destinados al funcionamiento de la institución académica (centros docentes y de investigación), al servicio de la misma (residencias, apartamentos estudiantes...) y en el marco de un nuevo espacio de Burgos polarizado en la idea del campus (viviendas), constituye la realidad que explica tanto las actuaciones de transformación como las de renovación. 
Figura 3. Transformación del conjunto tradicional denominado "Plaza del Sobrado".
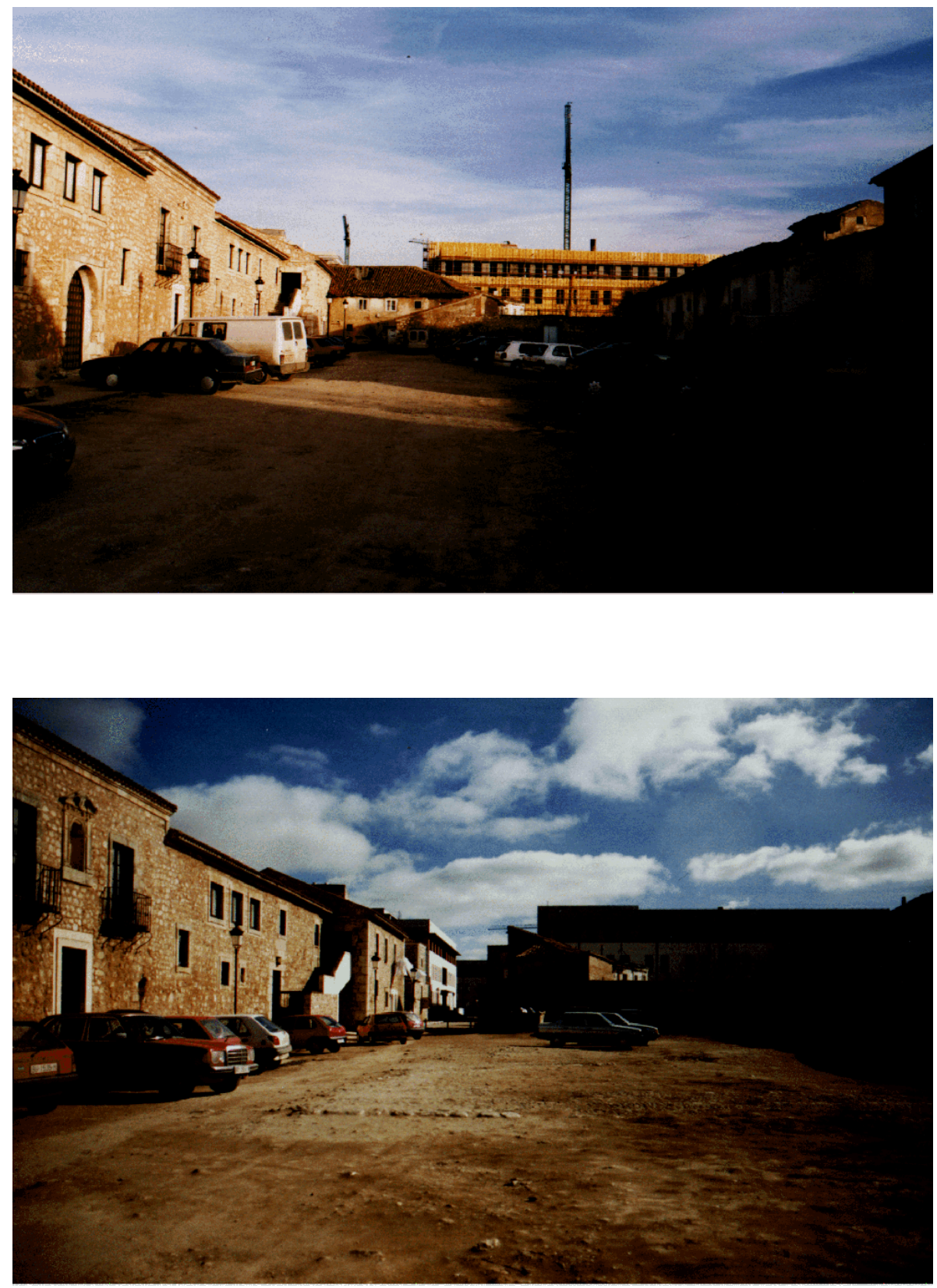
Ahora bien, podemos plantearnos numerosas incógnitas y diversos problemas respecto a esta nueva forma de entender el Suroeste. En primer lugar, Burgos no es la ciudad que desde el modelo universitario se ha pretendido entender, ni por lo que respecta a su tamaño, ni en función de su dinamismo económico. Se trata de un núcleo de tamaño medio en España y con una posición definida en la región castellanoleonesa, lo que la sitúa en un intervalo de posibilidades concreto. Cierto es que Burgos atesora uno de los mayores incrementos en el índice de inversión industrial durante la última década en relación con una vitalidad económica reconocida ${ }^{18}$, pero no es menos real el hecho de que no pase de ser un núcleo que no llega a los 170.000 habitantes y en el que las previsiones de desarrollo atribuidas a la función universitaria parecen estar sobredimensiona das.

La visión que tiene la Universidad del espacio destinado a consolidar su campus está centrada en torno a unas previsiones que dependen del notable crecimiento de Burgos cuando lo cierto es que, pese a su vitalidad económica, la ciudad tiende más bien a mantenerse que a crecer ${ }^{19}$. Hay que tener en cuenta que la gestión de un cambio urbano exige no sólo ideas originales sino medidas adecuadas a la realidad social y económica del núcleo. En este razonamiento cabría plantearse la idoneidad del modelo de campus único. No hay que olvidar que el concepto de campus surge en el siglo XIX en la Universidad inglesa con la necesidad de crear espacios autónomos y autosuficientes respecto a los congestionados núcleos urbanos. No parece ser esa la necesidad actual de la Universidad española, máxime cuando las deficiencias de los espacios universitarios segregados se han manifestado en casos como el de Madrid en los que el modelo tenía su explicación en virtud del tamaño de la ciudad (GómEZ MENDOZA, 1987).

La Universidad de Burgos ha justificado el modelo espacial escogido en función de una serie de razones diversas centradas en configurar un nuevo elemento urbano como hito y atractivo turístico que aproveche las potencialidades del entorno en el que se enmarca. Esta idea se ha acompañado de una filosofía prospectiva singular en la que, según los redactores del documento presentado por la UBU al Ayuntamiento de Burgos, "en los años 40 el abuelo vivía en el pueblo y se dedicaba al campo, a la agricultura y a la ganadería. En los años 60, 70 y 80, el hijo trabajaba y trabaja en las fábricas de los polígonos industriales de Burgos. En los años 90 el nieto está estudiando en la Universidad de Burgos. En los años 2020 los bisnietos trabajarán unos en las fábricas y otros, cada vez más, serán

\footnotetext{
${ }^{18}$ Entre 1984 y 1994 la ciudad de Burgos y los municipios del entorno con inversión industrial (Villalbilla y Villagonzalo Pedernales fundamentalmente) han concentrado casi el $30 \%$ del total de inversiones industriales de las capitales de Castilla y León manifestándose como el centro más dinámico de la región al recibir una inversión superior a los 14.000 millones de pesetas en ese periodo. MANero Miguel, F y Pascual Ruiz-VALDEPeÑas, H: «Castilla y León ante el cambio industrial: ajustes productivos y estrategias de desarrollo», en JUNTA DE CASTILLA Y LEÓN: La Economía de Castilla y León ante el siglo XXI, Valladolid, 1999, p 109 - 167.

${ }^{19}$ No debemos olvidar que entre 1991 y 1996 la provincia de Burgos ha perdido casi 3.000 habitantes. En el mismo periodo la ciudad ha pasado de tener 160.278 a 166.117 habitantes. Las proyecciones de población para los próximos diez años indican una tendencia al estancamiento de la provincia y un leve avance en la capital que, en ningún caso, superaría los 200.000 habitantes. LÓPEZ TRIGAL, L: «La población y el poblamiento en Castilla y León» en JUNTA DE CASTILLA Y LEÓN: La Economía de Castilla y León ante el siglo XXI, Valladolid, 1999, p 9 - 31.
} 
investigadores científicos y tecnológicos dedicados a la producción inmaterial en el Parque Tecnológico y Científico de San Amaro" ${ }^{20}$.

No cabe duda que estos principios prospectivos tienen un importante valor para la gestión y el dinamismo de una ciudad como Burgos pero la concreción de las actuaciones ha de tener en cuenta otras muchas dimensiones. La idea de entender el campus de San Amaro como un reclamo de Burgos para el exterior y consolidarlo como uno de los espacios turísticos del núcleo es sin duda una iniciativa a considerar pues esta ciudad necesita un gran proyecto que le dote de imagen con respecto al exterior. Sin embargo, esta necesidad no ha de ser ajena a los problemas de otros sectores de Burgos y, de hecho, ha de ser utilizada en su recuperación. Desde la propia Institución Académica, Bernal Santa Olalla ha demostrado en muchas ocasiones la conveniencia de recuperar el centro histórico y la necesidad de establecer una adecuación entre las nuevas actuaciones, la conservación del patrimonio y la revitalización de los espacios degradados ${ }^{21}$.

En esta dinámica, las tensiones producidas por la necesidad de conjugar los nuevos elementos urbanos y los espacios de mayor tradición histórica han aparecido sin remisión y no únicamente por lo que respecta a la función universitaria. La polémica suscitada por la nueva composición espacial del suroeste de la capital burgalesa en el entorno del Real Monasterio de las Huelgas no es sino un claro ejemplo de los problemas inherentes a este tipo de procesos ${ }^{22}$. Por un lado hay que valorar el interés histórico, artístico, social y popular del propio Monasterio declarado Bien de Interés Cultural y con una rancia participación en la conformación de este sector de la ciudad. Por otro, hay que tener en cuenta los intereses de un conjunto de propietarios que persiguen obtener rentabilidad económica de sus inversiones para la construcción de viviendas en el marco del nuevo desarrollo urbano de este espacio de la ciudad aglutinado en torno a la naciente Universidad.

Por encima de todo ello, debe primar el sentido de ciudad y el entendimiento

\footnotetext{
${ }^{20}$ Documentación complementaria a la Alegación formulada por la Universidad de Burgos en el trámite de Información pública del Documento de Revisión del Plan General de Ordenación Urbana de Burgos. Septiembre de 1997. Unidad Técnica de la Universidad de Burgos. p 16.

${ }^{21}$ A través de diversas publicaciones y trabajos de investigación, Begoña Bernal Santa Olalla ha demostrado la necesidad que Burgos tiene de conjugar las nuevas actuaciones urbanas reguladas en el nuevo planeamiento de la ciudad con el mantenimiento y la conservación funcional de los espacios históricos. De entre ellos, hay que destacar aquí, por las referencias al tema en el que se centra este artículo, los siguientes textos: «Relación Universidad - Ciudad en Burgos. Un posible compromiso con el patrimonio y la calidad urbana», en Bernal SANTA Olalla, B. (Coord.): Ciudad Histórica y Calidad Urbana. Seminario Vivir las Ciudades Históricas, Ed. Fundación La Caixa y Universidad de Burgos, Burgos, 1999, pp 21 - 42 y en Bernal Santa Olalla, B. (1999): «La descentralización del centro. El caso de Burgos», en Revitalización Funcional del Centro Histórico. Un reto de las ciudades históricas, Ed. Servicio de Publicaciones. Universidad de Burgos, p 45 -65 .

${ }^{22}$ Los problemas suscitados en torno a la construcción de viviendas en el perímetro del Monasterio de las Huelgas (Plan Parcial Parral y Sectores de Suelo Urbanizable 14 y 15) dibujan un claro ejemplo de las tensiones desatadas en los procesos urbanos en los que conviven la tradición y las nuevas implantaciones. Las parcelas aledañas a la huerta del Monasterio han reducido finalmente sus aprovechamientos delimitándose un entorno de protección y ajustándose la altura de los edificios más cercanos al cenobio. Plan General de Ordenación Urbana. Ayuntamiento de Burgos. Servicio de Urbanismo. Boletín Oficial de la Provincia de Burgos, Viernes 2 de Julio de 1999.
} 
racional de las actuaciones urbanas en un marco regulador impuesto por la ordenación urbana y la gestión local. En la definitiva aprobación del actual Plan General, parece haberse comprendido esta necesidad quedando delimitada la necesaria franja de protección para un espacio histórico tan singular como el compuesto por el Monasterio y sus fincas aledañas, aún a costa de la renuncia municipal a desarrollar los aprovechamientos públicos en beneficio de la promoción privada.

Ambos segmentos, el del interés particular y el de la regulación municipal, han convergido en el suroeste de Burgos aportando, en definitiva, una nueva composición espacial para este sector de la ciudad. Desde la posición colateral que nos ofrecen disciplinas como la geográfica hemos de cuestionarnos si la transformación y renovación que está afectando al suroeste de la capital burgalesa se rige por los parámetros adecuados o va a generar un espacio demasiado rígido y con una función muy concreta dentro del núcleo. El Parral, las Huelgas, el Hospital del Rey... han sido siempre lugares de la ciudad apreciados por la población, aun cuando su integración en el resto del núcleo fuese a costa de una serie de actividades marginales con respecto a la norma dominante. El suroeste de Burgos ha tenido tradicionalmente su función concreta y parece haberse entendido a lo largo de la historia como un espacio para el servicio colectivo de la ciudad.

Sin embargo, no es menos cierto que este sector ha manifestado con frecuencia los problemas inherentes a la marginalidad en el uso del suelo. La inseguridad ciudadana, el asentamiento de grupos sociales marginales, la degradación de algunos espacios... han sido un rasgo tan perteneciente al Suroeste como su entidad histórica vinculada al barrio de Huelgas o al Hospital del Rey. La renovación de usos aquí analizada supone una nueva función directora del devenir urbano, la de la Universidad, que, irreversiblemente, va a ser la nueva seña de identidad de este conjunto. De la actividad industrial, que tradicionalmente aportaba personalidad a este sector, se ha pasado a la enseñanza superior como elemento más importante del espacio urbano.

Su significado en Burgos ha de permanecer pues se trata, al fin y al cabo, de una sustitución de funciones en un espacio que sigue estando al servicio de la ciudad y que tiene un peso histórico muy fuerte. Ahora bien, los problemas de un uso específico, y la enseñanza universitaria lo es, no han desaparecido ${ }^{23}$. La creación de grandes unidades urbanas a través de edificios docentes reproduce la rigidez de los tradicionales usos industriales y genera porciones urbanas que propician una importante falta de articulación dadas sus dimensiones. A esto hay que sumarle el incremento de movimientos de tráfico diario que esta nueva actividad genera en un conjunto todavía no preparado para recibir importantes intensidades de vehículos dada su tradicional rigidez organizativa.

La clave de este proceso está, sin duda, en la población, en los propios habitantes ya que, a la postre, son estos los que dotan de vida al espacio urbano. Los nuevos ocupantes del Suroeste solucionarán, en parte, el problema de una única función dominante en el uso de este espacio. Pese a ello su integración con el resto de Burgos reclamará todavía una mayor accesibilidad y, de manera contemporánea,

23 "La Universidad vuelve a lanzar un Sos por los problemas de seguridad en el campus", Diario de Burgos, Martes 27 de Abril de 1999. 
la ciudad seguirá sin tener resuelto un grave conflicto como es el de la recuperación y revitalización del centro histórico.

\section{BIBLIOGRAFÍA.}

- Álvarez Mora, A. (1978): La remodelación del centro de Madrid, Ed. Ayuso, Madrid, 239 p.

- ANDRÉS LÓPEZ, G. (1999): «De las ordenanzas municipales al primer Plan de la democracia: origen y evolución del planeamiento urbano en la ciudad de Burgos», en Boletín de la Institución Fernán González, Burgos, 1999/2, pp 415450.

- ANDrÉs López, G. (2000): La Castellana. “Ciudad Jardín” en Burgos, Ed. Dossoles, Burgos, 270 p + 33 láminas cartografía.

- BERNAL SANTA OLALlA, B. (1999): «La descentralización del centro. El caso de Burgos», en Revitalización Funcional del Centro Histórico. Un reto de las ciudades históricas, Ed. Servicio de Publicaciones. Universidad de Burgos, p 4565.

- Bernal Santa Olalla, B. (1999): «Relación Universidad - Ciudad en Burgos. Un posible compromiso con el patrimonio y la calidad urbana», en Ciudad Histórica y Calidad Urbana. Seminario Vivir las Ciudades Históricas, Ed. Fundación La Caixa y Universidad de Burgos, Burgos, pp 21-42.

- GÓMEZ MENDOZA, J. Y OTROS (1987): Ghettos universitarios. El campus de la Universidad Autónoma de Madrid, Ed. Universidad Autónoma de Madrid, 196 p.

- GonzÁlez, N. (1958): Burgos, la ciudad marginal de Castilla. Estudio de Geografía Urbana, Aldecoa, Burgos, 307 p.

- López TRIGAL, L (1999): «La población y el poblamiento en Castilla y León» en JUNTA DE CASTILLA Y LEÓN: La Economía de Castilla y León ante el siglo $X X I$, Valladolid, p 9-31.

- MANERO Miguel, F. (1982): «La industria y las ciudades de Castilla La Vieja y León», en Actas del Primer Congreso de Geografía de Castilla la Vieja y León, Burgos, p $213-241$.

- MANero Miguel, F. y PASCuAl Ruiz-Valdepeñas, H (1999): «Castilla y León ante el cambio industrial: ajustes productivos y estrategias de desarrollo», en JUNTA DE CASTILLA Y LEÓN: La Economía de Castilla y León ante el siglo XXI, Valladolid, p 109-167.

RESUMEN: En los años noventa la ciudad española ha experimentado procesos de transformación urbana sumamente interesantes para la perspectiva de la Geografía al producirse la conjunción de cambios meramente físicos con la mutación social y funcional del espacio urbano. Estos hechos han coincidido en el caso concreto de la ciudad de Burgos donde el sector suroccidental del núcleo ha experimentado en los últimos quince años una transformación en la que la Universidad y los nuevos conjuntos de viviendas son los factores fundamentales de un proceso de cambio a través del cual se ha abandonado la tradicional orientación industrial del conjunto. El acercamiento a las pautas fundamenta- 
les de este proceso y su valoración por lo que respecta a la estructura urbana de una ciudad media como Burgos constituyen el objetivo más importante de este artículo.

PALABRAS CLAVE: Transformación urbana, cambio funcional, suroeste de Burgos, Universidad, vivienda.

ABSTRACT: In the nineties, the Spanish town has experimented extremely interesting processes of urban transformation from the Geographical standpoint, a collection of physical changes with social changes and functional ones in urban space. These actions have coincided in a specific case in the town of Burgos where the south-western sector of the nucleus has experimented in the last fifteen years a transformation in which the University and new housing blocks are the fundamental factors in a process of change through which the traditional industrial orientation of the area has been abandoned. The approach of the fundamental norms of this process and its assessment, in respect to the urban structure of an average sized city like Burgos, forms the essential objective of this paper.

KEY WORDS: Urban transformation, functional change, south-west Burgos, University, housing.

RÉSUMÉ: Pendant les années 90, las villes espagnoles ont eprouvé des processus de transformation urbaine très intéresants dès la perspective de la Geographie, par la liaison des changements simplement physiques et de la mutation social et fonctionnel de l'espace urbaine. Ces faits se sont rencontrés dans la ville de Burgos, où le secteur sud-ouest a souffert une transformation dans les derniers quinze ans. La Université et les nouveaux ensembles de logements sont les facteurs essentiels d'un processus de changement avec quoi la traditionnelle orientation industrielle a été abandonnée. Le rapprochement aux normes de ce processus et sa estimation par rapport à l'estructure urbaine d'une ville moyenne tel que Burgos constituent l'objectif de cet article.

MOTS-CLÉS: Transformation urbaine, changement fonctionnel, sud-ouest de Burgos, Université, logement. 